\title{
Analysis of the plastic zone of a circle crack under very high cycle fatigue
}

\author{
Tieying $\mathrm{Wu}^{\mathrm{a}, *}$, Danièle Wagner ${ }^{\mathrm{b}}$, Daniel Kirk ${ }^{\mathrm{c}}$ \\ ${ }^{a}$ Nanjing University of Aeronautics and Astronautics, College of Energy and Power Engineering, Nanjing 210016, PR China \\ ${ }^{\mathrm{b}}$ LEME, EA4416, Université Paris Ouest, 50, rue de Sévres, 92410 Ville d'Avray, France \\ ${ }^{\mathrm{c}}$ Florida Institute of Technology, 150 W, University Blvd, Melbourne, FL 32901, USA
}

Keywords:

VHCF

Inverse heat transfer method

Stress intensity factor

Plastic zone

\begin{abstract}
A B S T R A C T
Metals used in industry for structures and aero-engine components are sometimes subjected to very high cycle fatigue (VHCF) damage during their working service. In this paper, a novel method is presented to determine the size and location of a circular crack located within a metal specimen under $20 \mathrm{kHz} \mathrm{VHCF}$ loading conditions. The method is based on an analysis of the temperature rise on the surface of the specimen and correlation of this temperature rise to the energy dissipation in the plastic zone of the crack. The approach taken is to first determine the heat source location and strength using an inverse heat transfer calculation based on the surface temperature measurements. Next, the relationship between the heat and the material hysteresis loop in the plastic zone, which is a function of stress intensity factor and vibration amplitude cyclic loading of the specimen, is found. The calculation of the stress intensity factor under vibrational loading is often an obstacle in VHCF research because there is currently no standard or existing formula. In this paper a general polynomial formula for the stress intensity factory under $20 \mathrm{kHz}$ loading conditions is obtained using a finite element modeling approach as a function of the specimen's material properties and position and size of the internal crack.
\end{abstract}

\section{Introduction}

In the early 1990s, Bathias [1] demonstrated that metals fail due to very high cycle fatigue (VHCF) when subjected to more than $10^{10}$ cycles. Many materials, including some steels and cast irons, exhibit a sharp decrease in fatigue strength when subjected to between $10^{6}$ and $10^{10}$ cycles. This behavior is in contrast to the classical fatigue strength limit, below which a material is assumed to have an infinite life. Current theories are not able to properly describe the degradation of a material's fatigue strength in the VHCF regime, and the prediction of material properties and component lifetimes are often determined from experimentation.

Internal friction produced within a material during cyclic loading results in the generation of several energy forms including elastic strain energy, plastic strain energy, and inelasticity and heat generation and dissipation [2-4]. Ultrasonic experiments have demonstrated that energy generation and dissipation in the VHCF regime results in an increase of temperature of the test specimen. Even in the cases of confined plasticity or micro plasticity, the heat generation and dissipation is not negligible. The heat generated and transmitted within the material during high cyclic loading

\footnotetext{
* Corresponding author.

E-mail address: tieying.wu@nuaa.edu.cn (T. Wu).
}

depends on a number of factors including mechanical deformation, stress amplitude, and loading frequency. In other words, VHCF damage depends on complicated energy dissipation processes that are not yet fully understood.

For materials with inclusions, the crack initiation site may be internal to the specimen and acts as a heat source within the material during high cyclic loading conditions. The dissipated energy (DE) from the internal crack site can be identified by the temperature evolution of the specimen. The heat generated from internal circular cracks results in a temperature field on the surface of a specimen called a "fish eye formation". Fish eye formations have been observed during VHCF loading experiments when the number of cycles increases beyond $10^{7}$ cycles Bathias [1]. The model developed by Paris for fish eye formation in the gigacycle fatigue regime demonstrated that crack initiation takes a very long time after which crack propagation quickly occurs over a small number of cycles $[1,2]$.

There is an interesting link between crack formation and growth and the DE from the internal crack site. A promising approach is to measure the specimen's surface temperature field, which is directly related to the DE at the crack site, and then to use this information to identify and characterize the internal crack's size and location. In this way, the heat dissipation and the evolving temperature field of the material can be excellent 
parameters by which to better follow and understand VHCF damage. Several earlier works have demonstrated that the fish eye formations observed on the surface of a specimen are typical damage indicators associated with crack initiation in VHCF [1,3-6].

\section{Stress intensity factor calculation approach}

The stress intensity factor (SIF) is often used to characterize and quantify the influence of cracks in metals. However, there is no standard formula for the SIF in VHCF cases. In VHCF loading cases with excitations frequencies in the $20-30 \mathrm{kHz}$ range, the inertial force, which is proportional to the frequency squared, can be very high. Under these conditions, it is incorrect to apply standard static formulas of SIF to VHCF because the inertial forces cannot be ignored [5].

Fig. 1 shows an example of a tensile test specimen with a cylindrical diameter of $\Phi=10 \mathrm{~mm}$ and a neck diameter of $\Phi=3 \mathrm{~mm}$. The specimen has a constant diameter cylindrical length of $L_{1}$ on each side of the neck region, and the transition length between the constant diameter section and the minimum cross-sectional area at the neck is given by $\mathrm{L}_{2}$. The specimen has a circular internal crack of radius $r$ located within the neck region and $R$ is the eccentric distance of the crack center from the centerline of the specimen.

Using the geometry shown in Fig. 1, a finite element method (FEM) computational model was developed to calculate the SIF associated with a circular crack under VHCF loading conditions. A quarter of the specimen cut from the half circular crack is meshed for FEM analysis. Singular elements, in which the stress is theoretically infinite, are used at the tip of the crack. SIF calculations performed in this manner are very sensitive to the boundary conditions and size of the singular elements [5]. In this case, the boundary condition is that all nodes in the cut surfaces except those on the crack surface are applied only by null perpendicular displacement. The size of the singular elements that were used in the FEM model are made the smallest allowed in ANSYS software in consideration of SIF definition in fracture mechanics.

In the FEM simulation the specimen is subjected to axial tension-compression loading. The following equation was used in FEM calculation [1,5] to solve vibration modals:

$[K]\{u\}=\omega^{2}[M]\{u\}$

where $[K],[M]$ are rigidity and mass matrix respectively, $\{u\}$ is displacement vector. $\omega=2 \pi f, f$ is intrinsic frequency of the specimen. The SIF can be obtained from $\{u\}[5]$ and is proportional to the vibration amplitude, $A_{0}$, measured by a displacement sensor at the end of the specimen.

At room temperature, Eq. (1) can be modified to:

$\left[K^{\prime}\right]\{u\}=\left(\omega \sqrt{\frac{\rho}{E}}\right)^{2}\left[M^{\prime}\right]\{u\}$

where $\left[K^{\prime}\right]$ and $\left[M^{\prime}\right]$ depend only on specimen geometry and $\sqrt{\frac{E}{\rho}}$ is the longitudinal wave propagation speed of the material, Fortunately, this value is around $5 \mathrm{~km} / \mathrm{s}$ for many typical metallic alloys, such as aluminum, titanium, nickel based alloys, and steels. For the specimen shown in Fig. 1, the length $L_{1}$ should be calculated to keep its intrinsic frequency at $20 \mathrm{kHz}$ by following functional relationship shown in Eq. (3) which was obtained from FEM calculations:

$L_{1}=2217.46-2.257 \sqrt{\frac{E}{\rho}}-254.26\left(\sqrt{\frac{E}{\rho}}\right)^{2}+33.34\left(\sqrt{\frac{E}{\rho}}\right)^{3}$

For a circular crack, the SIF can vary along the circumference of the crack while under vibrational loading. In one position on the
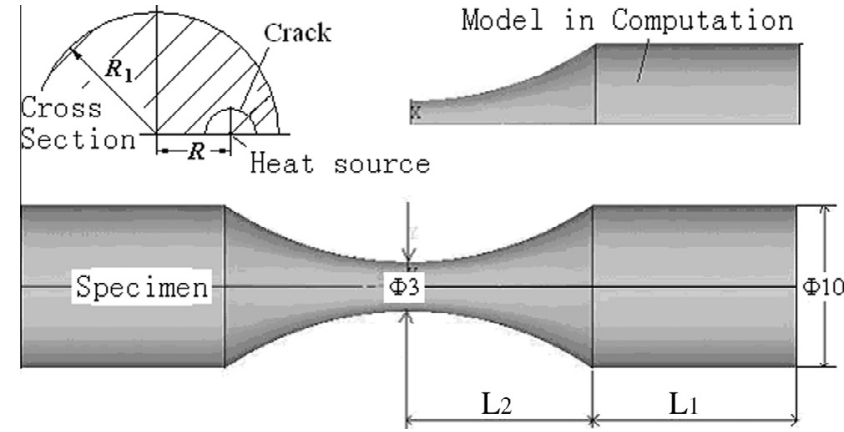

Fig. 1. Tensile test specimen with circular crack located in the neck.

circumference, the stress intensity factor, $K_{I}$, can be considered as plane strain, described by following function:

$K_{I}=\frac{\mathrm{E}}{1-v^{2}} \sqrt{\frac{\pi}{r}} A_{0} f\left(\frac{r}{R_{1}}, \frac{R}{R_{1}}\right)$

Here, E, $v$ are Young's module and Poisson's ratio. $f\left(\frac{r}{R_{1}}, \frac{R}{R_{1}}\right)$ represents the geometric factor, which is a function of 2 dimensionless ratios associated with the size, $r / R_{1}$, and position, $R / R_{1}$, of the crack.

According to FEM calculations, $K_{\mathrm{I}}$ does not vary much along the circle when the crack is small and in-body. For example, consider the case of a large crack located very close to the surface of the specimen with $r=0.4 \mathrm{~mm}$ and $R=0.9 \mathrm{~mm}$. In this case the variation of $K_{I}$ along the circumference of the crack is less than $8 \%$. In fracture surfaces, a fish-eye with an inclusion in the center can be seen and it proves this result.

For a given specimen and material, values of the geometric factor function $f$ can be found through FEM calculation by varying $r$ and $R$. After applying the least square method, formula (5) is obtained and function (4) presents average SIF in the circular crack:

$$
\begin{aligned}
f\left(\frac{r}{R_{1}}, \frac{R}{R_{1}}\right)= & -0.169\left(\frac{r}{R_{1}}\right)+8.25\left(\frac{r}{R_{1}}\right)^{2}-66.14\left(\frac{r}{R_{1}}\right)^{3} \\
& +145.9\left(\frac{r}{R_{1}}\right)^{4}+1.22\left(\frac{r R}{R_{1}^{2}}\right)-32.23\left(\frac{r^{2} R}{R_{1}^{3}}\right) \\
& +243.8\left(\frac{r^{3} R}{R_{1}^{4}}\right)-526.9\left(\frac{r^{4} R}{R_{1}^{5}}\right)-1.02\left(\frac{r R^{2}}{R_{1}^{3}}\right) \\
& +26.63\left(\frac{r^{2} R^{2}}{R_{1}^{4}}\right)-192.6\left(\frac{r^{3} R^{2}}{R_{1}^{5}}\right)+404.2\left(\frac{r^{4} R^{2}}{R_{1}^{6}}\right)
\end{aligned}
$$

In addition, Fig. 2 shows that values of $f$ calculated by FEM are almost the same for a given crack location (crack located on the centerline of the specimen at $\mathrm{R}=0$ ) but different metals. This is the same result as found in previous studies [5].

Eqs. (4) and (5) can be used for any metallic specimen having the same geometry shown in Fig. 1 and $20 \mathrm{kHz}$ intrinsic frequency.

\section{Fatigue dissipated energy and crack tip plastic zone analysis}

This section develops an analysis to determine the volume and total deformation energy associated with the plastic zone at the tip of a crack under VHCF loading conditions. In-body crack initiations have been located from defect-like non-metallic inclusions, microfissures and microstructural defects (residual austenite, bainite and ferrite). Fish-eye shapes are always present in the internal failures, almost a perfect circle, located with distance between 0.2 and $1 \mathrm{~mm}$ from a surface. 


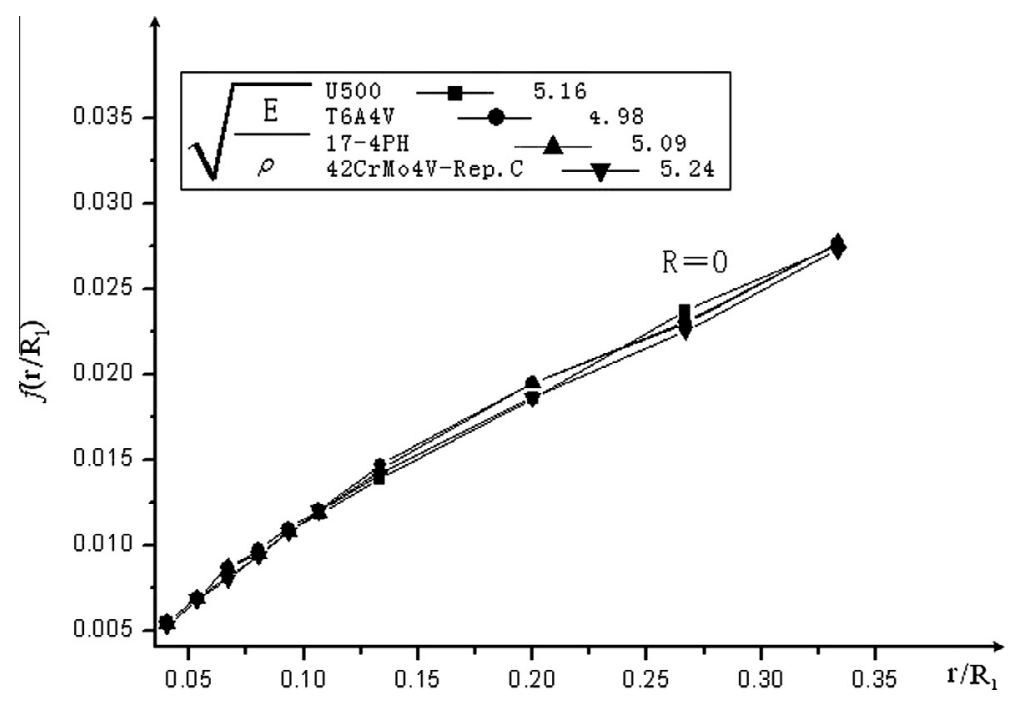

Fig. 2. Geometrical factor of $K_{I}$ defined as a function of crack size for different metals.

\subsection{Analysis of the volume of the plastic zone at the crack tip}

The deformation mechanisms associated with the plastic zone at the tip of an internal crack can be explained through a comparative study of the form and dimensions associated with the plastic zone. The form of the plastic zone depends not only on the state of stresses but also on the size of the involved crack. Analysis of the effective plastic stress at the crack tip requires applying VonMisses criteria to determine the size and volume of the plastic zone, as shown in Eq. (6):

$$
\sqrt{\frac{1}{2}\left[\left(\sigma_{1}-\sigma_{2}\right)^{2}+\left(\sigma_{2}-\sigma_{3}\right)^{2}+\left(\sigma_{3}-\sigma_{1}\right)^{2}\right]}=\sigma_{y}
$$

where $\sigma_{1}, \sigma_{2}$, and $\sigma_{3}$ are the three principal stresses, and $\sigma_{\mathrm{y}}$ is the yield stress of the material obtained during uniaxial tension. Yielding occurs when the differences of stresses on the left side of the Eq. (6) exceed the yield stress in uniaxial tension, $\sigma_{y}$.

In the VHCF regime the crack within the specimen propagates from the inside to the outside of the specimen. For a specimen used in $\mathrm{VHCF}$, there is a plane-strain condition. Thus, plane-strain values of critical stress results in the plastic deformation at the crack tip. In other words, a plastic zone will exist at the crack tip. Here three principal stresses are given as:

$$
\begin{aligned}
& \left.\begin{array}{l}
\sigma_{1} \\
\sigma_{2}
\end{array}\right\}=\frac{K_{I}}{\sqrt{2 \pi r_{1}}} \cos \frac{\theta_{1}}{2}\left(1 \pm \sin \frac{\theta_{1}}{2}\right) \\
& \sigma_{3}=\sigma_{z z}=v\left(\sigma_{1}+\sigma_{2}\right)=2 v \frac{K_{I}}{\sqrt{2 \pi r_{1}}} \cos \frac{\theta_{1}}{2}
\end{aligned}
$$

Here, $r_{1}$ is the distance from the center of the crack, $\sigma_{z z}$ is one of the principal stresses, and $\theta_{1}$ is the angle from the $\mathrm{x}$-axis (see Fig. 3 below). The size of the plastic zone is explained in fracture mechanics:

$r_{1}=\left(\frac{K_{I}^{2}}{2 \pi \sigma_{y}^{2}}\right) \cos ^{2} \frac{\theta_{1}}{2}\left[(1-2 v)^{2}+3 \sin ^{2} \frac{\theta_{1}}{2}\right]$

Function (9) presents the dashed line in Fig. 3, the tip of the crack is in fact just one point in the circular crack tip.

By combining Eqs. (6)-(9), and assuming that the Poisson's ratio is equal to 0.3 , the volume of the plastic zone can be calculated by integrating around the circumference of the circular crack, as shown in Eq. (10):

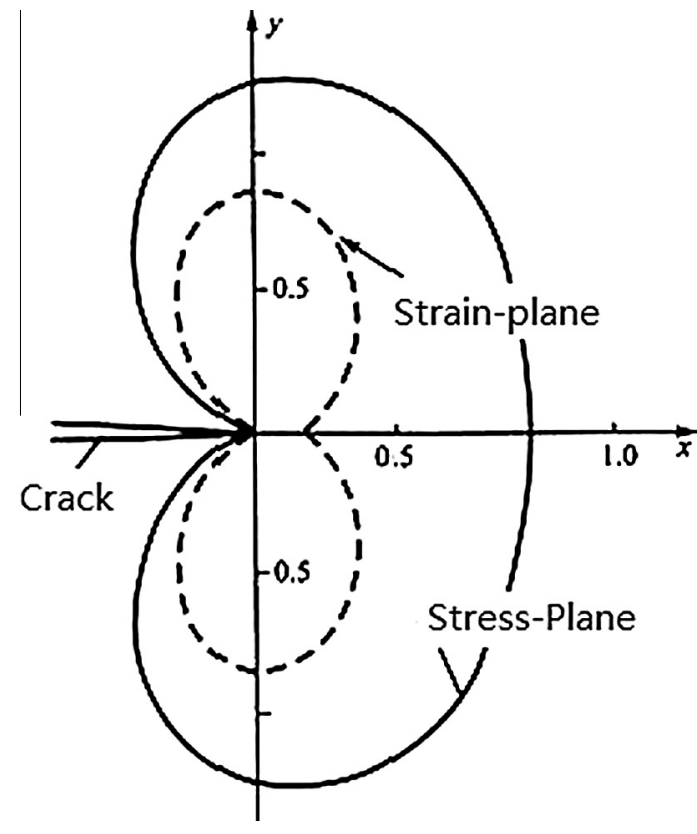

Fig. 3. Plastic zone shape at tip of a crack.

$$
\begin{aligned}
V & =2 \int_{0}^{\pi} \int_{0}^{\cos ^{2} \frac{\theta_{1}}{2}\left(0.16+3 \sin ^{2} \frac{\theta_{1}}{2}\right)\left(\frac{K_{I}^{2}}{2 \pi \sigma_{y}^{2}}\right)} 2 \pi\left(r+r_{1} \cos \theta_{1}\right) r_{1} d r_{1} d \theta_{1} \\
& =6.895 \times 10^{-4} K_{I}^{6} \sigma_{y}^{-6}+1.4 \times 10^{-1} r K_{I}^{4} \sigma_{y}^{-4}
\end{aligned}
$$

where $K_{\mathrm{I}}$ is maximum value during the constant amplitude vibration and can be calculated by using Eq. (4). Eq. (10) demonstrates that the plastic volume grows with increasing $K_{\mathrm{I}}$ and creases in volume with decreasing with $\sigma_{\mathrm{y}}$.

\subsection{Analysis of the total deformation energy of the crack tip from the stress-strain loop}

When a material enters into the plastic regime under cyclic loading the deformation energy can be calculated through the fatigue stress-strain (hysteresis) loop shown in the left of Fig. 4.

The situation is irreversible and the deformation energy is converted to heat. This loop can be approximated as a parallel 


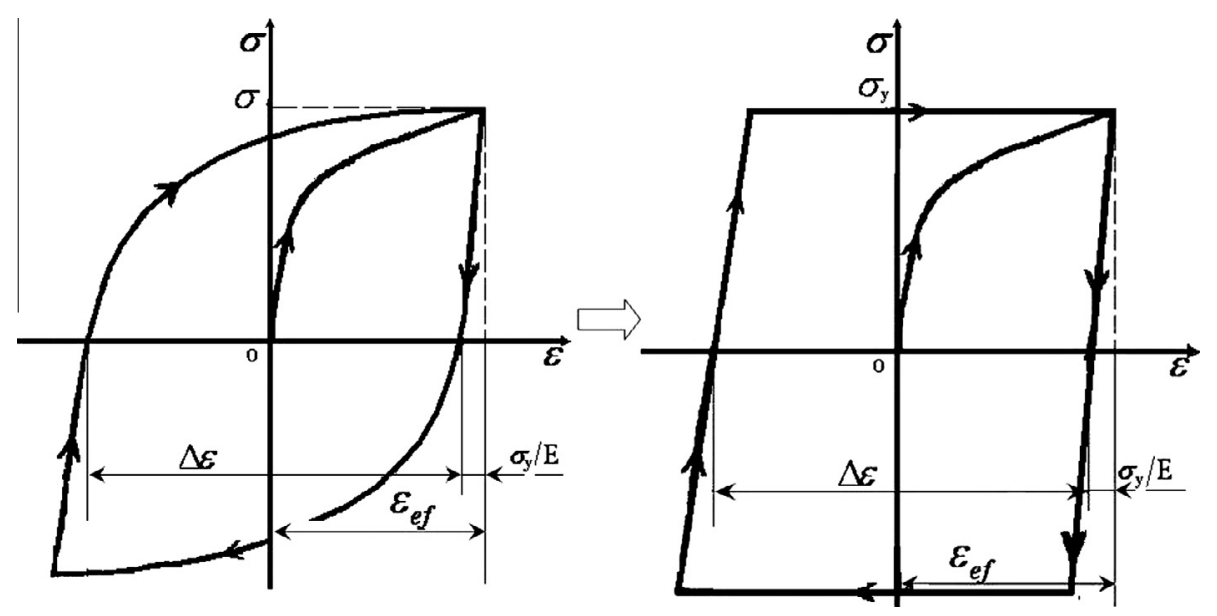

Fig. 4. Stress-strain loop under monocycle for constant strain cycling.

quadrilateral as shown in the right illustration of Fig. 4. The width of the hysteresis loop depends on the level of cyclic strain. The area of this quadrilateral in tension-compression loading is equal to:

$u \approx 2 \sigma_{y} \Delta \varepsilon=4 \sigma_{y}\left(\varepsilon_{e f}-\frac{\sigma_{y}}{\mathrm{E}}\right)$

This equation demonstrates that the specific strain energy, or absorbed energy per unit volume, of the material as a result of crack tip plasticity is represented by the area surrounded by $\sigma$ vs. $\varepsilon$ loop. This loop can be shown schematically by tracing $\sigma$ vs. $\varepsilon$ points generated during cyclic oscillation, where the strain passes from a minimum to a maximum and then arrives to a minimum by ignoring strain hardening. Under cyclic loading, the appearance of a crack in the specimen results in the passing of the stored strain energy beyond the threshold value. Under very high frequency $(20-30 \mathrm{kHz})$ loading, the storage of the strain energy is very rapid and induces failure very rapidly. Under these conditions the growth time associated with the crack can be ignored $[1,6]$.

The generalized form of Hooke's law can be used to determine the relationship between principal strains with principal stresses $[3,7]$, as shown in Eq. (12):

$$
\left.\begin{array}{l}
\varepsilon_{1}=\frac{1}{\mathrm{E}}\left[\sigma_{1}-v\left(\sigma_{2}+\sigma_{3}\right)\right]=\frac{K_{l}}{\mathrm{E} \sqrt{2 \pi r_{1}}} \cos \frac{\theta_{1}}{2}\left(0.52+1.3 \sin \frac{\theta_{1}}{2}\right) \\
\varepsilon_{2}=\frac{1}{\mathrm{E}}\left[\sigma_{2}-v\left(\sigma_{1}+\sigma_{3}\right)\right]=\frac{K_{l}}{\mathrm{E} \sqrt{2 \pi r_{1}}} \cos \frac{\theta_{1}}{2}\left(0.52-1.3 \sin \frac{\theta_{1}}{2}\right) \\
\varepsilon_{3}=\frac{1}{\mathrm{E}}\left[\sigma_{3}-v\left(\sigma_{1}+\sigma_{2}\right)\right]=0
\end{array}\right\}
$$

The effective strain (Von Mises Law) can be assigned by 3 principal strains, as shown in Eq. (13):

$$
\begin{aligned}
\varepsilon_{e f} & =\frac{\sqrt{2}}{3} \sqrt{\left(\varepsilon_{1}-\varepsilon_{2}\right)^{2}+\left(\varepsilon_{2}-\varepsilon_{3}\right)^{2}+\left(\varepsilon_{3}-\varepsilon_{1}\right)^{2}} \\
& =\frac{2 K_{I}}{3 \mathrm{E} \sqrt{2 \pi r_{1}}} \cos \frac{\theta_{1}}{2} \sqrt{0.27+5.07 \sin ^{2} \frac{\theta_{1}}{2}}
\end{aligned}
$$

Eq. (13) can be integrated over the total plastic zone, and the total plastic strain energy, $U$, due to plastic zone for one cycle can be calculated as follows:

$$
\begin{aligned}
& U=\iiint u d v \\
& =2 \int_{0}^{\pi} \int_{0}^{\cos ^{2} \frac{\theta_{1}}{2}\left(0.16+3 \sin ^{2} \frac{\theta_{1}}{2}\right)\left(\frac{K_{l}^{2}}{2 \pi \sigma_{y}^{2}}\right)} 4 \sigma_{y}\left(\varepsilon_{e f}-\frac{\sigma_{y}}{\mathrm{E}}\right) 2 \pi\left(r+r_{1} \cos \theta_{1}\right) r_{1} d r_{1} d \theta_{1} \\
& =\sigma_{y}^{-4} \mathrm{E}^{-1}\left(6.4 \times 10^{-1} r \sigma_{y}^{2} K_{I}^{6}-4.65 \times 10^{-3} K_{I}^{6}\right)
\end{aligned}
$$

As an example, consider a crack with radius $\mathrm{r}=0.08 \mathrm{~mm}$ located within three materials with different values of $\sigma_{\mathrm{y}}$ and E. Eq. (14) can be used to show the evolution of plastic strain energy, $U$, as a function of $K_{\mathrm{I}}$ under the condition of maximum displacement during vibration. The results of this calculation are shown in the Fig. 5 for aluminum, titanium, and nickel alloys.

Most of this irreversible deformation energy will become heat and will be dissipated to the outside of the specimen and manifested as an increase in temperature. Some researchers have estimated that around $90 \%$ of the irreversible deformation energy is transformed to heat [8]. Fig. 5 demonstrates that the plastic strain energy, $U$, for constant $K_{I}$ and constant crack radius, $r$, is higher for materials with low Young's Modulus, $E$, and low yield stress, $\sigma_{y}$.

\section{Identifying crack location based on the observed surface temperature field}

The heat generated at the crack during VHCF loading applications depends on material properties, the size of the crack, and the stress intensity factor, $K_{\mathrm{I}} . K_{\mathrm{I}}$ depends on material properties, crack size and location, and the vibration amplitude. Among these parameters, material properties and the vibration amplitude are known, but crack size and location are typically unknown. However, if the temperature field on the surface of the specimen can be recorded during testing, then an inverse heat transfer method (IHTM) can be used to locate the position and density of the responsible heat source [9]. A modern and commonly used IHTM is the neural network approach. The neural network is taught by a set of results based on normal heat transfer methods: from source to temperature. In this paper, a database (DB) method is developed and employed.

\subsection{Database modeling}

Database methods are widely used, and the most popular commercial DB is the relational DB, which is based on relational algebra used to implement its database management system (DBMS). Any DB platform can be used such as MySQL. According to the specific application, data can be modeled as Entities and Relations (ER model) to insure data integrity and security. Fig. 6 gives the ER model for this approach. There are 2 tables: CASE and DATA. DATA describes related node coordinates, temperature, temperature gradient and timestamp.

For the cases presented in this work, the timestamp is not used because it is impossible to synchronize the start time in inverse problem as related to the direct solution cases. Without time, tem- 


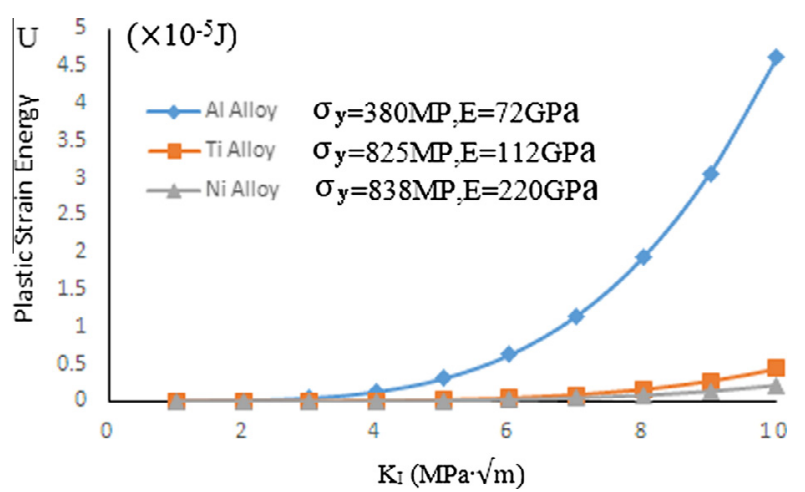

Fig. 5. Evolution of plastic strain energy, $U$, as a function of stress intensity factor, $K_{\mathrm{I}}$.

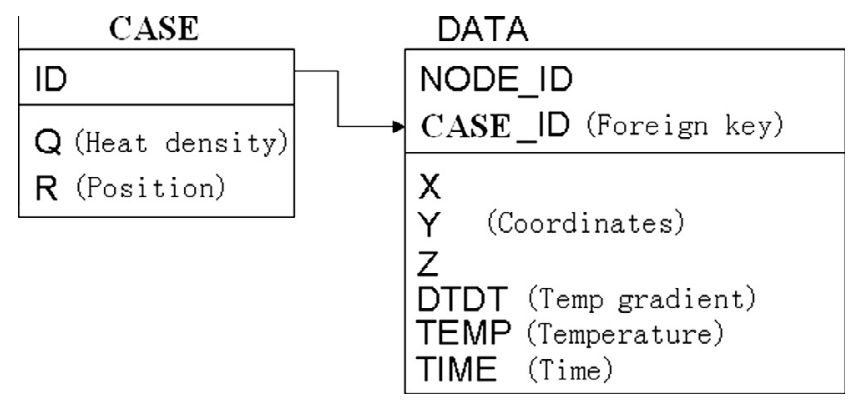

Fig. 6. ER model in database.

perature also becomes insignificant. From the curve in Fig. 2, the slope (temperature gradient) is a good characteristic. Theoretically, the temperature gradient is a term of the basic heat transfer equation. This is the reason that DTDT (dT/dt) must be one of attributes of node data.

\subsection{Data calculation from direct method}

For the heat transfer calculation associated with the specimen shown in Fig. 1, the distance $R$ between heat source position and the center of the specimen is one of the givens, as well as the heat density per cycle, $Q$ (watt). The computed cases are characterized by $R$ and heat density $Q$. After meshing the specimen, setting initial and boundary conditions including environment $\left(20^{\circ} \mathrm{C}\right.$ and convective heat transfer coefficient $\left.120 \mathrm{~W} /\left(\mathrm{m}^{2}{ }^{\circ} \mathrm{C}\right)\right)$, all the cases are calculated for a given steel specimen. Q was varied from $0.25 \mathrm{~W}$ to $5.0 \mathrm{~W}$ in increments of $0.25 \mathrm{~W}$ ( 20 values of $\mathrm{Q})$ and $\mathrm{R}$ was varied from $0 \mathrm{~mm}$ to $1.425 \mathrm{~mm}$ in increments of $0.074 \mathrm{~mm}$ (20 values of $R)$. There are 400 combinations of different $Q$ and $R$ that were analyzed using the software. In the results, the software outputs temperature and its gradient at each node and at each time. There are 441 surface nodes, 40 time-steps for one case. For one case, the output data presents the evolution of temperature on surface of the specimen with time. 400 data sets make more than 7 million records imported into the database table.

\subsection{Inverse problem solution}

When the direct solution results are ready, the inverse solution can be done based on the recorded temperature data. Some points on specimen surface can be chosen according to exact node coordinates associated with the calculation to minimize error. The highest temperature point should be one of them and at least one other point in the same section.

Following SQL query can be sent to the DB to get a set of data:

SELECT Q, R, count $\left(^{*}\right)$

FROM CASE A, DATA B

WHERE A.ID = B.CASE_ID AND

$($ NODE_ID $=: 1$ AND DTDT $<: 2$ AND DTDT $>: 3)$ AND

GROUP BY Q, R

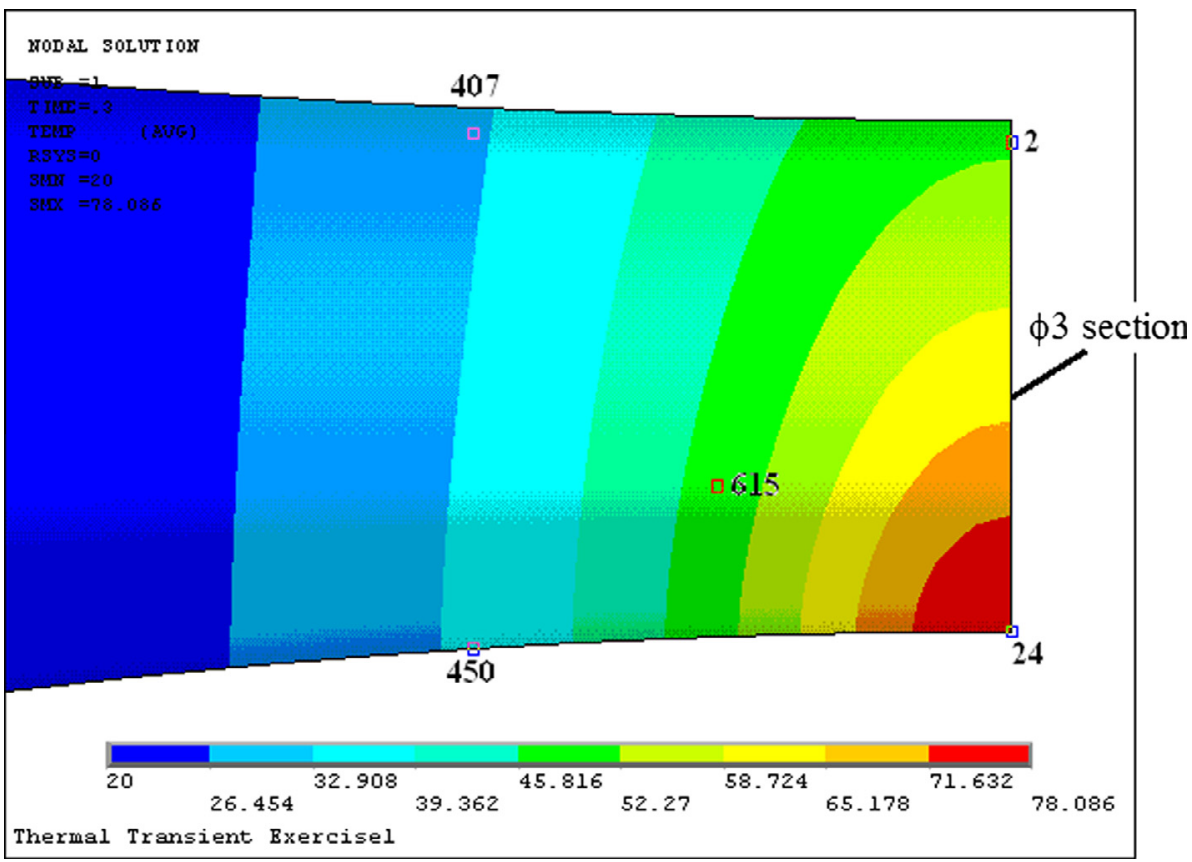

Fig. 7. Temperature field and 5 measured points in the specimen. 
Table 1

Simulated measurement values.

\begin{tabular}{|c|c|c|c|c|c|}
\hline NODE & $\mathrm{X}(\mathrm{mm})$ & $\mathrm{Y}(\mathrm{mm})$ & $\mathrm{Z}(\mathrm{mm})$ & TEMP $\left({ }^{\circ} \mathrm{C}\right)$ & $\mathrm{dT} / \mathrm{dt}\left({ }^{\circ} \mathrm{C} / \mathrm{s}\right)$ \\
\hline 2 & 0 & 1.5 & 0 & 48.00 & 79.09 \\
\hline 24 & 0 & 0 & -1.5 & 74.00 & 81.84 \\
\hline 407 & 2.26 & 1.582 & 0 & 29.85 & 49.89 \\
\hline 450 & 2.26 & 0 & -1.5823 & 31.70 & 50.82 \\
\hline 615 & 1.23 & 0.832 & -1.2772 & 45.19 & 70.03 \\
\hline
\end{tabular}

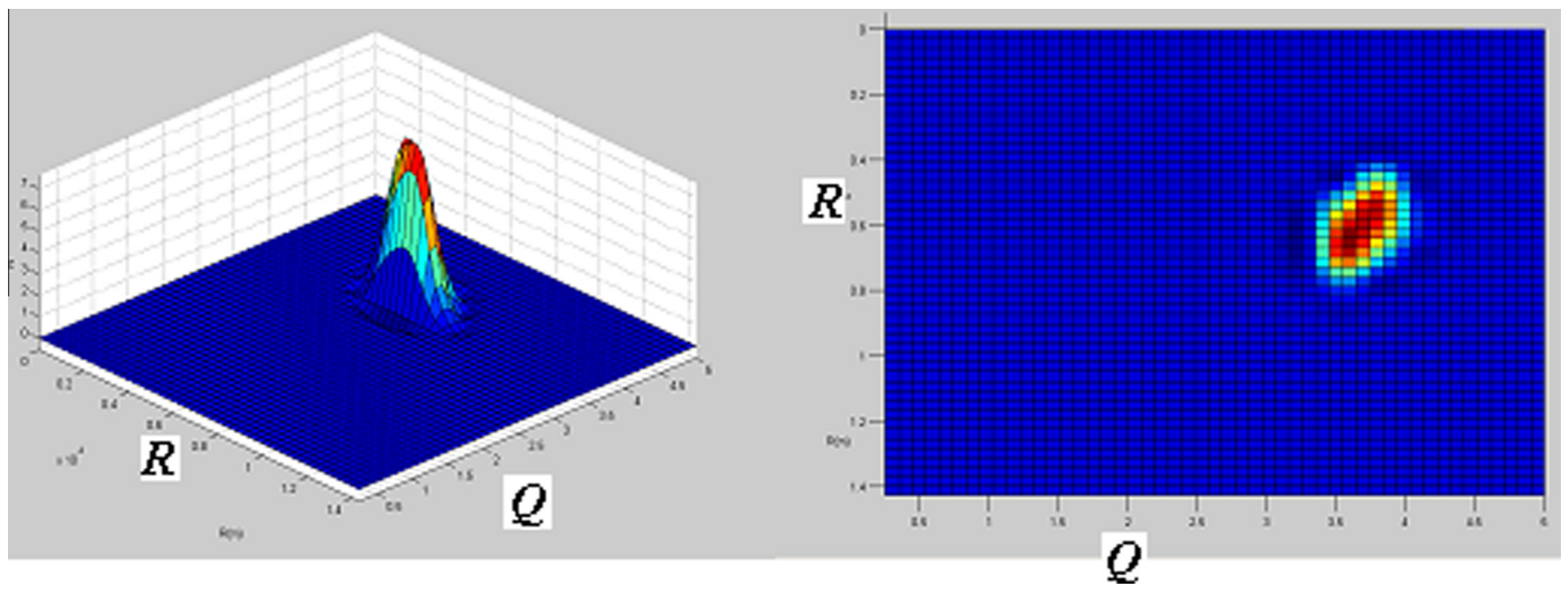

Fig. 8. Simulation result by DB query.

Table 2

Test case results.

\begin{tabular}{|c|c|c|c|c|c|}
\hline & True value & Average & Error1 & Peak & Error2 \\
\hline $\mathrm{Q}(\mathrm{W})$ & 3.6 & 3.61818 & $0.505 \%$ & 3.5459 & $-1.5 \%$ \\
\hline $\mathrm{R}(\mathrm{mm})$ & 0.273 & 0.23 & $-43 \mu \mathrm{m}$ & 0.29082 & $17.82 \mu \mathrm{m}$ \\
\hline Q2 (W) & 3.6 & 3.63 & $0.83 \%$ & 3.5459 & $-1.5 \%$ \\
\hline $\mathrm{R} 2(\mathrm{~mm})$ & 0.613 & 0.592 & $-21 \mu \mathrm{m}$ & 0.639 & $26 \mu \mathrm{m}$ \\
\hline Q3 (W) & 3.6 & 3.621951 & $0.61 \%$ & 3.5459 & $-1.5 \%$ \\
\hline $\mathrm{R} 3(\mathrm{~mm})$ & 1.2273 & 1.22 & $-7.3 \mu \mathrm{m}$ & 1.1923 & $-35 \mu \mathrm{m}$ \\
\hline
\end{tabular}

Basically, this SQL joins two tables together by CASE_ID and all the predicates are AND conditions. In one pair of parentheses, the first predicate at :1 defines a measured node position. Parameters $: 2$ and :3 defines a temperature gradient range at this node. The parentheses may be replicated with different values according to number of measured points. After sending this SQL to the DB, it will return a set of data which combine $Q, R$ and number of appearance of the case. With several different measured points, different sets of data can be retrieved. From this SQL statement, several cases may be returned, i.e. those cases satisfy the given surface "symptom". However, the total number of appearance of a case means sensitivity, more or less, of the case to the surface measurement values.

When the DB data model is correctly designed, the response time of this instruction takes only a fraction of second. For this example specimen, importing only the data in wasp waist part may reduce number of records in the database and speed the SQL query.

The selected measured point position is very important as it will impact directly the selectivity of the SQL statement. In addition, the number of measured points also has influence on the solution. Generally, the more points there are the better the selectivity.

Some examples have been calculated by the using the software to verify this method. For the purpose of generality, a case is calculated with $Q=2.8 \mathrm{~W}$ and $R=0.13636 \mathrm{~mm}$, which is not an exact match of one of the 400 combinations of $Q$ and $R$ described earlier (i.e. this exact combination is not represented in the DB). 5 measured points are chosen as shown in Fig. 7 and Table 1 lists the simulated measurement values.

By putting those values into the SQL statement and sending it to the DB, returned results are presented in Fig. 8 where the vertical coordinate in left figure is the count of case. There is apparently a peak, meaning the most sensible combination of $R$ and $Q$ estimated solution. $R$ and $Q$ in the peak are very close to the known values. It can be seen that many cases do not appear in this query (blue $^{1}$ part), that is to say, much less data interference with this solution.

Table 2 lists some simulation examples where $Q=3.6 \mathrm{~W}$ and 3 different $R$ 's. 2 methods have been tried for the estimation of the solution. One is just to make weighted average all case values returned by $\mathrm{DB}$; another is the peak estimation.

In general, the weighted average method is better than peak estimation due to the positions of nodes 2 and 24 (see Fig. 7). Estimation error is smaller when the heat source position is close to the surface (see Fig. 9) since the "symptom" appears clearer and more significant on those 2 nodes.

\footnotetext{
${ }^{1}$ For interpretation of color in Fig. 8, the reader is referred to the web version of this article
} 


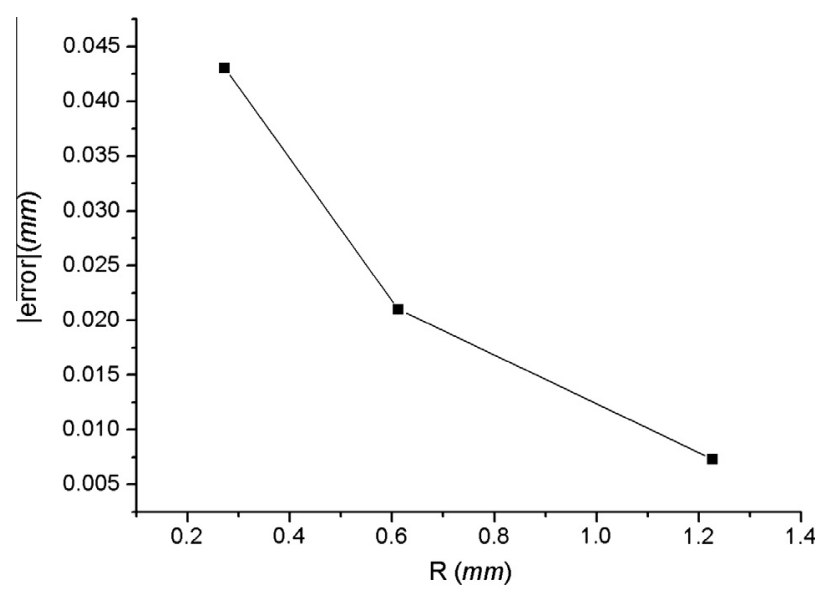

Fig. 9. Position estimation error vs. position.

\section{Conclusions}

In this paper, an analytical approach was developed and validated to calculate the stress intensity factor, $K_{\mathrm{I}}$, for the specimen specially designed for VHCF test. Calculation modeling is in 3D, for in-body circle crack under vibration mode. Formula (4) and (5) are suitable for metal specimen of any materials. Calculated variation of $K_{\mathrm{I}}$ around the circle is not significant and can be neglected.

According to fracture mechanics, volume of plastic zone at tip of the crack depends on $K_{\mathrm{I}}$. Formula (12) gives plastic deformation energy in the plastic zone per cycle under fatigue for symmetric loading. $90 \%$ of the energy power dissipates as heat observed by infrared camera.

Combining the database inverse method and the relationship between energy power and SIF, determination of crack size and position from temperature field on the specimen surface become possible.

\section{Acknowledgement}

Supported by "the Fundamental Research Funds for the Central Universities”, NO. NZ2016103.

\section{References}

[1] Bathias C, Paris PC. Gigacycle fatigue in mechanical practice. Marcel Decker Inc.; 2005. ISBN 0-8247-2313-9.

[2] Ranc N, Wagner D, Paris P. Study of thermal effects associated with crack propagation during very high cycle fatigue tests. Acta Mater 2008;56 (15):4012-21.

[3] Ranc N, Paulin-Luc T, Paris P. Thermal effect of plastic dissipation at the crack tip on the stress intensity factor under cyclic loading. Eng Fract Mech 2011;78:961-72.

[4] Ranc N, Paulin-Luc T, Paris P, Staintier N. About the effect of plastic dissipation in heat at the crack tip on the stress intensity factor under cyclic loading. Int J Fatigue 2014:58:56-65.

[5] Wu T, Bathias C. Application of fracture mechanics concepts in ultrasonic fatigue. Eng Fract Mech 1994;47(5).

[6] Bayraktar E, Garcia MI, Bathias C. Failure mechanisms of automotive metallic alloys in very high cycle fatigue range. Int J Fatigue 2006;28(11):1590-602.

[7] Paris PC. Crack initiation mechanism in very high cycle fatigue. In: Proceedings of the VHCF-3 conference, Kyoto, Japan.

[8] Huang Z, Wagner D, Bathias C. Subsurface crack initiation and propagation mechanisms in gigacycle fatigue. Acta Mater 2010;58:6046-54.

[9] Chang M. Development and applications of inverse heat transfer method. VDM Verlag; 2009. ISBN 3639140931. 